e-migrinter

\section{e-Migrinter}

$9 \mid 2012$

Immigrés, illégaux, réfugiés. Questions sur les enquêtes et les catégories

\title{
Expériences migratoires
}

Cycle de séminaires conjoint IRIS-EHESS et MIGRINTER, 2011-2012

\section{Céline Bergeon et Caroline Rozenholc}

\section{OpenEdition \\ Journals}

Édition électronique

URL : https://journals.openedition.org/e-migrinter/782

DOI : 10.4000/e-migrinter.782

ISSN : 1961-9685

Éditeur

UMR 7301 - Migrinter

Édition imprimée

Date de publication : 20 juillet 2012

Pagination : 107-109

ISSN : 1961-9685

Référence électronique

Céline Bergeon et Caroline Rozenholc, «Expériences migratoires », e-Migrinter [En ligne], 9 | 2012, mis en ligne le, consulté le 20 mai 2021. URL : http://journals.openedition.org/e-migrinter/782 ; DOI :

https://doi.org/10.4000/e-migrinter.782 


\title{
Cycle de séminaires sur les expériences migratoires ; Paris, IRIS-EHESS ; Poitiers, MIGRINTER, 2011-2012
}

\author{
Compte-rendu par Céline Bergeon \& Caroline Rozenholc
}

$\mathbf{P}$

our la seconde année consécutive, Marie-Antoinette Hily et Yann Scioldo-Zürcher (Migrinter) organisaient avec Michèle Leclerc-Olive (IRISEHESS) un cycle de séminaires consacré aux « expériences migratoires ». Cette année, c'est la question de la fragmentation et de

la narrativité de l'expérience migratoire qui était au cœur des échanges. En privilégiant une approche pluridisciplinaire, l'objectif de ces séances était de discuter le décalage, la dissonance, entre une expérience de la migration le plus souvent faite d'arrangements, de discontinuités et de ruptures, et sa retranscription habituellement linéaire. À cet effet, les travaux des chercheurs présentés durant ce cycle - ceux de chercheurs confirmés, jeunes chercheurs et doctorants - se sont appuyés sur plusieurs formes "d'écriture de la migration ": des Systèmes d'Informations Géographiques à la littérature, en passant par le cinéma.
Au fil des séances, les interventions ont permis de mettre en lumière les difficultés à retranscrire les émotions et les expériences vécues par les migrants, les nomades, les exilés ou les personnes déplacées dans la diversité de leurs trajectoires. Dans cet esprit, la présente note restitue trois communications qui font toutes appel à des outils et des matériaux différents pour retranscrire le déplacement et narrer la migration.

« L'utilisation d'un Système d'Information Géographique pour l'étude de la mobilité tsigane dans le Sud-Ouest français dans les années 1920 », le 3 février 2012

[Adèle Sutre, Doctorante en Histoire à l'EHESS (Paris)]

Lors de la première journée de ce cycle de séminaires, Adèle Sutre, doctorante à l'EHESS, a abordé la question de la retranscription des données d'archives en matériaux cartographiques. Son travail de thèse s'appuie, en effet, sur les carnets anthropométriques que les populations tsiganes étaient tenues de porter, en France de 1912 à 1969, pour retracer et cartographier certains de leurs itinéraires. Durant cette période, et avec la promulgation de la loi du 16 juillet 1912, les Tsiganes, considérés comme des «nomades", étaient astreint au port de ce carnet qu'ils devaient faire viser lors de leurs déplacements et notamment, à chaque arrivée et départ d'une commune française. Ainsi, une base de données qu'Adèle Sutre a 
constituée en relevant l'ensemble des lieux précisés par les visas, lui a permis de retracer les itinéraires de nombreuses familles tsiganes au cours des années 1920. Les traitements cartographiques de ces données ont alors montré des continuités dans les itinéraires et les routes empruntées par ces familles. Elle précise néanmoins que le recours à ce genre de matériau nécessite une connaissance précise de la population étudiée et des discours qui lui sont attachés pour en faire l'interprétation cartographique. Ainsi, par exemple, la plupart des itinéraires reconstitués dans le travail cartographique d'Adèle Sutre suivent le chemin des cours d'eau du Sud-Ouest français. Cette observation peut s'expliquer par l'exercice de l'activité de vannier, très courante chez les tsiganes de la région et qui nécessite la présence d'eau pour le travail de l'osier. Néanmoins, outre cette explication «rationnelle» aux trajets effectués par les familles, d'autres motivations, qui échappent totalement au cartographe, ne peuvent être exclues. C'est d'ailleurs l'une des interrogations de Yann Scioldo-Zürcher qui, en historien, pointe les difficultés à organiser et restituer l'expérience du passé tout en évitant les pièges de la fixation de la narration: cette expérience n'est pas toujours visible dans les archives et autres sources historiques, d'où la difficulté à retranscrire les bifurcations dans les expériences de l'espace.

\section{«"Jours et temps d'exil" : migrations politiques et contraintes ", le 6 avril 2012}

[Sylvie Aprile, Professeur en histoire contemporaine, Université de Lille]

Dans son intervention, Sylvie Aprile, Professeur en histoire contemporaine à l'Université de Lille 3, a proposé une analyse des temporalités dans les récits de l'exil. Elle a particulièrement développé l'exemple des Proscrits du Second Empire à partir d'un matériau constitué de récits de la vie quotidienne, de romans, ou encore de témoignages d'auteurs célèbres. D'autres récits ont été rassemblés et traités à partir de publications américaines des années 18501860 ; tous retranscrivent le vécu de la migration des Proscrits. À partir de ce corpus, Sylvie Aprile a particulièrement insisté sur les fragmentations et les discontinuités présentes dans ces récits: fragmentations et discontinuités elles-mêmes amplifiées par une tendance à l'extension du temps à l'intérieur de ces témoignages de l'événement traumatique qui précisent la narration. Ce matériau permet donc aux chercheurs de faire un réel retour sur une expérience passée, en même temps qu'il opère une stabilisation du passé par le récit. Une interrogation émerge alors quant à l'absence, quasi systématique, du présent dans les écrits. Pourquoi le présent est-il si peu investi dans ces textes?

Sylvie Aprile a alors expliqué que les proscrits du Second Empire sont, pour la plupart, des exilés volontaires et/ou des exilés par condamnation; l'exil étant la peine la plus élevée. Ils sont majoritairement originaires de Belgique et de GrandeBretagne et aboutissent souvent en Amérique. La violence de cette situation d'exil introduit un rapport à l'écriture particulier pour les Proscrits, qui est d'abord un rapport à l'identité. L'ensemble des textes travaillés par l'historienne révèle une déperdition de la personnalité engendrée par l'exil et le déracinement. Elle montre que l'écrit a alors pour vocation de maintenir une présence et de perpétuer le nom de l'exilé. L'utilisation du passé et du futur permet alors à ce dernier de retranscrire l'expérience de l'exil et, dans une certaine mesure, de résister à la violence de son présent. Par ailleurs, ne pas écrire sur le présent, cela permet également de ne pas en ancrer la réalité. Ainsi, «le Proscrit se déplace dans le passé et le futur » avec une écriture du passé qui encadre et cristallise la mémoire, comme c'est le cas dans les récits de Victor Hugo. 


\section{« La construction d'un Grand Récit unifié de l'immigration arménienne en Éthiopie : modalités de réécriture et enjeux sociaux "}

[Boris Adjémian, docteur en histoire, EHESS - Université de Naples]

Dans sa présentation, Boris Adjémian, docteur en histoire de l'EHESS et de l'Université de Naples "L'Orientale», retrace l'histoire de l'immigration arménienne en Éthiopie, une immigration qu'il qualifie «d'originale et d'insaisissable » et dont les traces ont pour objectif principal d'historiciser l'installation des Arméniens en Éthiopie. Ces traces, qu'elles soient sous forme de biographies ou d'images, montrent une grande diversité des parcours, des origines sociales et culturelles des Arméniens présents en Éthiopie. Si les migrants arméniens se considèrent comme faisant partie intégrante de la société éthiopienne, leur installation dans le pays n'en est pas moins complètement absente de l'historiographie locale. À ce propos, il faut noter le peu de sources disponibles et le petit nombre d'Arméniens toujours présents en Éthiopie: aujourd'hui, ils ne seraient plus que 83. Les "autobiographies collectives» tendent à se diffuser au sein de cette population. L'histoire de la communauté est exposée de manière orale. Elle se cristallise sous la forme d'un grand récit et fonctionne comme un dialogue entre les descendants de migrants arméniens et la diversité des situations. Partant de là, l'analyse de ces récits laisse entrevoir deux phases importantes: celle du règne de l'Empereur Ménélik II et celle succédant à la guerre durant laquelle la diaspora arménienne se constitue. Au delà de l'étude de cas, cet exemple interroge la manière dont la mémoire collective fait le lien entre des expériences migratoires et le sentiment d'appartenance à une communauté. Pour illustrer son propos, Boris Adjémian raconte l'histoire de la création, en 1924, d'une fanfare royale, à Jérusalem, par le ras Täfäri (plus connu ensuite sous le nom d'Hailé Sélassié), composée de quarante enfants arméniens. C’est un événement historiquement très peu connu et qui peut, de fait, être interprété de diverses manières. Ces enfants, tous orphelins, ne sont jamais présentés comme des musiciens professionnels, mais uniquement comme des enfants adoptés. Pour B. Adjémian, il s'agit d'un « dialogue reconstitué » où s'opère une simplification profonde du récit dont sont supprimés tous les détails sur les conditions de voyage des enfants avant leur arrivée en Éthiopie. Dans ce récit, on note surtout la prégnance du devoir de loyauté envers le roi et la protection reçue en retour de cette allégeance. L'historien parle d'une réelle euphémisation de l'événement. Comment expliquer que le mythe de l'adoption ait fonctionné et comment expliquer, encore, le choix, par un roi éthiopien, d'immigrants arméniens? Ils peuvent être interprétés comme un acte politique qui fait jouer à l'Église chrétienne orthodoxe un rôle important. En effet, dans une période charnière de l'affirmation d'une souveraineté éthiopienne, le pouvoir politique exploite, par la constitution de cette fanfare " arménienne », les liens religieux exclusifs et les contacts séculaires entre les Églises éthiopienne et arménienne à Jérusalem.

\section{Conclusion}

Ce cycle de séminaires sur les expériences migratoires a exposé des supports d'analyse multiples, pour une critique de la retranscription du fait migratoire comme expérience vécue. Si le chercheur a souvent tendance à "détemporaliser les choses» pour exposer les faits qu'il tente de comprendre, ces séminaires ont néanmoins permis de déconstruire et d'analyser les usages du «montage narratif de soi », pour reprendre respectivement les propos de Michèle Leclerc-Olive et de Marie-Antoinette Hily. Le chercheur n'est-il pas abusé par ce narratif ? Comment peut-il rendre compte de 
ces/ses expériences? Faut-il effacer les discontinuités ou sinon, quelles places peuton leur donner dans la conduite d'une recherche scientifique? En conclusion, les organisateurs de ce cycle de séminaires rappellent que, comme l'a remarqué le philosophe Jacques Rancière dans nombre de ses ouvrages, le travail d'exposition de l'expérience se doit d'être couplé à celui de la retranscription: le chercheur doit continuellement prendre en compte la manière dont les fragments sont assemblés par celui qui raconte son histoire. Cela dit, faut-il faire entendre la parole, la voix du migrant, ou celle du chercheur en ce sens qu’il interprète les récits qu’il recueille?

Ce cycle de séminaires sur la thématique de la narration de l'expérience migratoire sera poursuivi et approfondi en 2012-2013, par des questionnements qui seront précisés et renouvelés par d'autres modalités d'expériences migratoires et matériaux de retranscription.

Argumentaire et programme 2011-2012 :

http://www.ehess.fr/fr/enseignement/enseignements/2011/ue/830/

Argumentaire et programme 2012-2013 :

http://www.ehess.fr/fr/enseignement/enseignements/2012/ue/777/ 